\title{
New Reliability Routing Path for Detects Malicious Link
}

\author{
Bulla Premamayudu $^{1 *}$, Leela Priya Inturu ${ }^{1}$, Gajula Ramesh ${ }^{2}$ \\ ${ }^{1}$ Vignan's Foundation for Science, Technology \& Research (Deemed to be university), Vadlamudi, Guntur, India \\ ${ }^{2}$ CSE Department, GRIET, Bachupally, Hyderabad, India
}

Corresponding Author Email: premamayudu@gmail.com

https://doi.org/10.18280/isi.240211

Received: 20 January 2019

Accepted: 5 April 2019

\section{Keywords:}

security, wormhole, most limited way, mobile specially appointed systems, applications, assaults, secure, binary search probing, reliability

\begin{abstract}
The custom of correspondence security conventions at first progressed for wire line and $\mathrm{Wi}-\mathrm{Fi}$ systems is available with substantial weight on the constrained system assets of a MANET. We propose new validating hubs is correspondence organize based on idea of shared trust. The propose calculation is find verifies and most brief way against wormhole assault. Directing portable specially appointed system is testing dynamic topologies. There are bunches of trust models and directing convention which are utilized in MANETs security. A versatile testing procedure recognizes a pernicious connection through twofold inquiry and as per the hubs conduct these connections are maintained a strategic distance from in the dynamic way by multiplicatively expanding their loads. Among these the security is the pinnacle issue looked by a large portion of the remote systems. The examination work proposes a system that identifies the particular sending assaults and figures the unsafe hosts living an impromptu structure. Recreation considers are directed utilizing NS2 to demonstrate that proposed methodology upgrade arrange execution when organize size, load or the portability increments.
\end{abstract}

\section{INTRODUCTION}

Specially appointed system is considered as a standout amongst the most rising innovations in this day and age. In an impromptu system, the hosts depend upon each other to empower and keep up the whole system conveying and connected together [1]. The convention shields pair insightful correspondence over an obscure often changing system plot displayed in this paper ensure that a byzantine flaw is recognized and the shortcoming connection can be stayed away from in the information transmission stage [2].

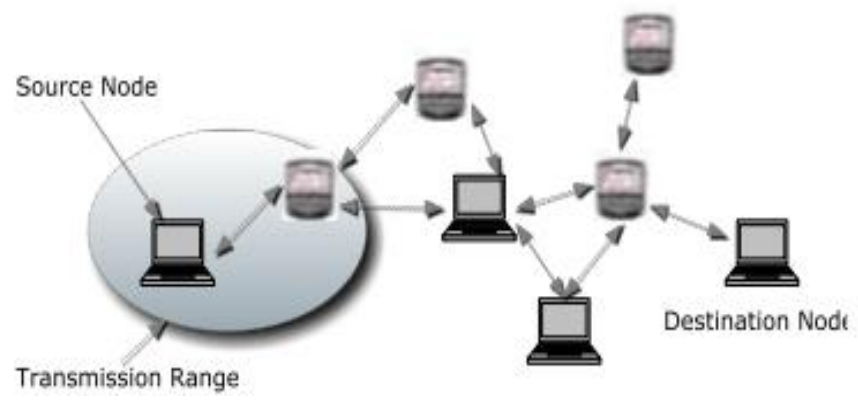

Figure 1. Mobile Ad-hoc network

Different assaults can be decreased because of the nearness of security conventions [3]. Distinctive conventions are then assessed dependent on parcel drop rate, overhead presented by steering convention security issue looked by the directing convention is thought about [4]. Proactive methodologies, for example, cryptography and verification and numerous different procedures is proposed and actualized these applications are not adequate. This can leave MANETs open to a scope of assaults, for example, the Sybil assault and course direction assaults that can mollify the uprightness of the system [5]. The work detailed in this paper address the steering issues of available directing convention in condition of MANETs and the directing presentation in testing condition of MANETs [6]. The below Figure 1 illustrates the MANET. The source node will send the data to other nodes in transmission range. The Destination node will receive that data via the available route. The below Figure1 illustrates the mobile ad hoc network structure.

\section{RELATED WORK}

Since the appearance of MANETs, structure and usage of a productive directing convention with great execution and less overhead is one of the major difficulties of this system [7]. This paper is expected to help scientists in building up their very own on-request specially appointed directing conventions and advancing clients in affecting the business plan that best meets their requirements [8].

The diverse parts of proposing security models in MANETs to identify with trust can be found in data innovation as trust measurements and trust assessment are essentially characterized for open key verification to get to control and electronic business [9].

Hierarchical directing and geographic position helped steering the expansion in adaptability can be accomplished by decreasing the quantity of rebroadcasting hubs [10]. In certain occasions we have to keep the data mystery from the majority of the unapproved hub or due to this might be malignant hubs and can hinder or annihilate the data.

So we need to keep up the secret data from the unapproved substance [11]. The message and the excess are separated into various pieces, so that even a halfway gathering can recreate 
the information called as Message scattering source refreshes the evaluations of the ways dependent on the criticism [12].

The safe key administration plot depended on the edge cryptography conspire worked effectively when it needed to convey in vast dispersed territories A reviving plan was utilized to counter the portable hub enemies [13]. The Figure2 Explains the process of message dispersion in SMT where communication is done between source and destination. The message dispersion in SMT process is shown in Figure 2.

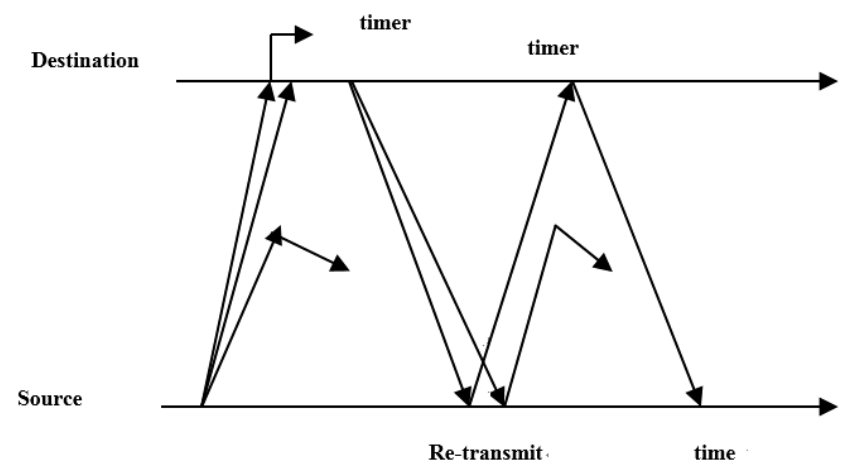

Figure 2. Message dispersion in SMT

\section{SYSTEM ARCHITECTURE}

The half breed steering convention is qualities of both responsive and proactive directing conventions is presented the overheads proportion and the underlying course revelation deferrals of existing directing conventions [14]. These interior assaults now and again may communicate wrong sort of steering data to different hubs inside assaults are noxious hubs that are a piece of the system, inward assaults are harder to identify than the outer assaults [15].

Flag Stability Based Adaptive (SSA) and AssociativelyBased Routing (ABR) conventions propose two distinct instruments for surveying join solidness. To maintain a strategic distance from such cases the choice factor utilizes a worldwide unique limit an incentive for certification the hub to remain in the correspondence generally leave the system [16]. The new proposed strategy is spine directing way is overhead and devour more transmission capacity and hubs control in correspondence diverse landscapes present separate difficulties to steering in high unique condition of MANETs [17].

\section{PROPOSED SCHEME}

The security information transmission is expanded choosing most verified courses in Active Path Set (APS) to improve the execution of the verified message transmission most dependable ways is chosen and incorporated into dynamic way set APS is components is given select the most solid ways [18]. The course demand is sent bounce by jump and computerized marks are utilized at each jump to keep a foe from indicating a self-assertive way course disclosure stage comprises of the accompanying stages. The overall view of the proposed method is shown in Figure 3.

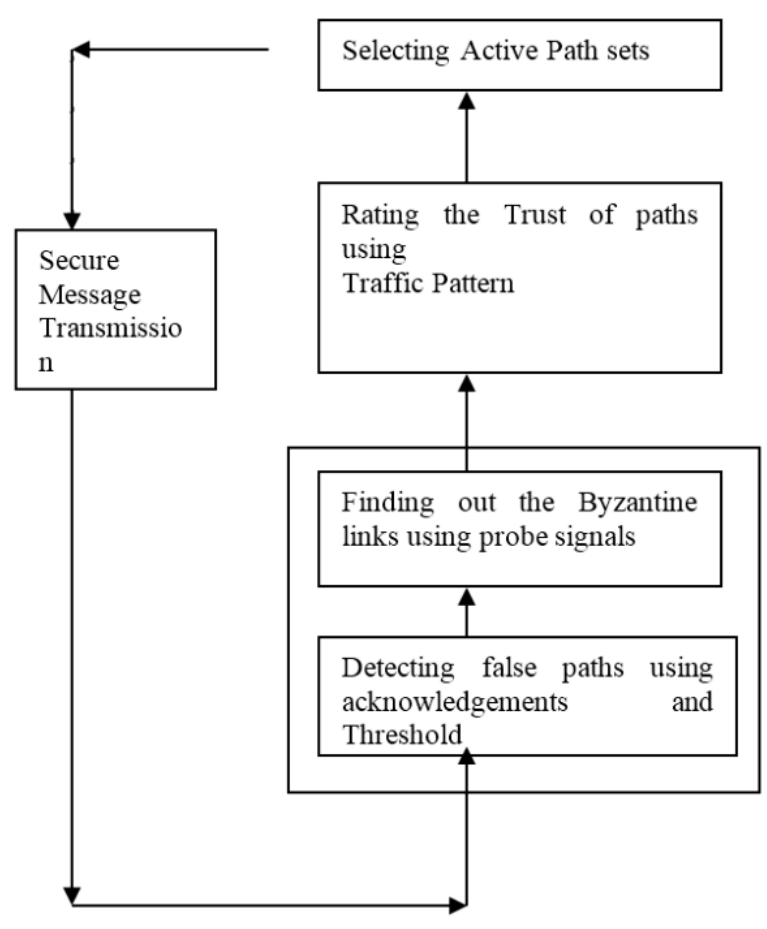

Figure 3. Overall view of proposed system

\subsection{Security algorithm}

The zone is little the hubs thought to be less malevolent movement made by the wormhole assault will be checked and the vindictive hub will be secluded the possibility of briefest way calculation is probabilistic technique for examination will be concentrated to cryptographic investigation will be made to keep running in an ongoing situation utilizing a continuous working framework.

The hash chain is executed on every parcel to make the correspondence secure. The control parcel is sent with every datum bundle. There are three instances of control ACK; a) positive control ACK, b) negative control ACK and, c) no control ACK c Control ACK" presents the three instances of control ACK.

Algorithm: Retrieve hash field value algorithm

Step-1: if(Final-Hash = F Hop Count ( Hash)) then

Step-2: Retrieve the packet count value in the Hash filed of the control packet.

Step-3: else

Step-4: Drop the control packet.

\subsection{Key management}

We are taking multi-hubs in our framework each host has complex ways to achieve a solitary end hub in the system [23]. The Daffier-Hellman key-trade methodology offers a method for making symmetric key age conceivable and is pushed off to make the Symmetric Keys (SK) keys. Symmetric Broadcast keys $(\mathrm{SKb})$ must be created by methods for a calculation that produces irregular number of relating secured key age administration [24]. This is cultivated utilizing a hashing calculation, for example, HMAC. Subsequently the bundle's adventure from point-to-point until the goal is come to is businesslike. 
Input: NODES, TA, PUBKEY,PRIKEY

Step-1: Node is provided with a certificate TA

Step-2: The joining node A seeks to join a network by periodically broadcasting discovery request packets containing its public Diffie-Hellmen Key share (DKSp). This continues and it recieves a certificate request from a networkable node B.

Step-3: A sens its certificate Exchange packet to B.

Step-4: B checks the integrity and authenticity of the certificate exchane (CEx) packet, using the shared $\mathrm{SK}_{\mathrm{p}}$.

Step-5: if certificate is deemed A is added to B's Security table. If the certificate fails this check, the credentials generated for node A by B is dropped and B and the process ends.

Step-6: if B has not authenticated any other nodes, it will generate $\mathrm{SKb}$ to the joining node.

Step-7: If A has a broadcast key, it transmits before table updation.

Step-8: B broadcast an SK invalidation packets with nodes within the network.

\section{RESULTS}

The examinations substantiate that the proposed framework is effectively adapt to a high number of enemies, Active Path Set Secure Message Transmission is conveyed numerous bundles effectively than Non-Secure Protocol is fruitful start to finish delay. System throughput is diminished as there is a nearness of malevolent hubs in pernicious hub hinders with the correspondence held between the hubs of the system the throughput of the system drops. the malignant hubs, their parcels are never again coasting in the system in addition to there is additionally no compelling reason to resend the dropped bundle henceforth expanding the general execution and decreasing the overhead of the system. The system inclusion is a $500 \mathrm{~m}$ by $500 \mathrm{~m}$ with 50 versatile hubs, with any two hubs ready to impart on the off chance that they are inside the gathering separation which is set to $150 \mathrm{~m}$. The performance of the NSP process is illustrated in Figure 4.

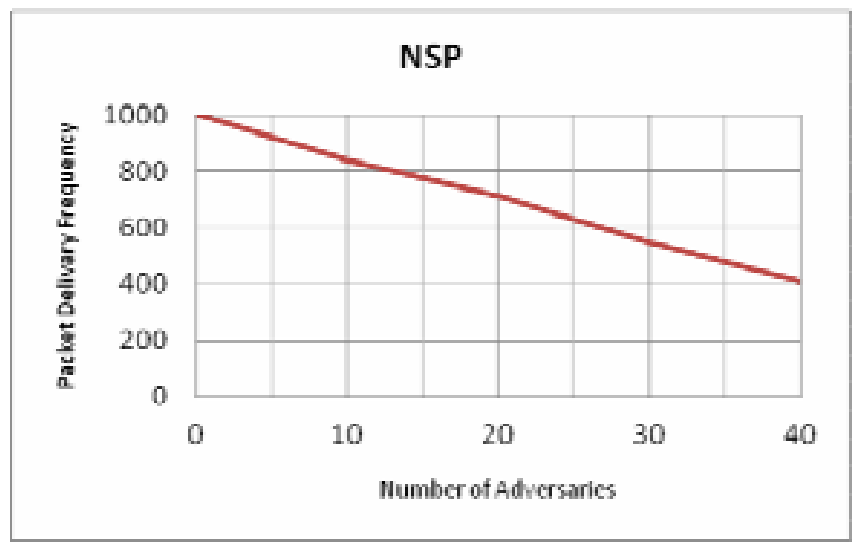

Figure 4. Performance of NSP

\section{CONCLUSION AND FUTURE WORK}

The work is figured to a progressed scientific idea to stretch out to a Wide territory Network. Our proposition is circulated powerful and does not depend itself on any focal system to coordinate association among hubs in the system is considered as an amount of involvement. These are named dynamic and latent assaults are attempted to actualize security calculation alongside directing conventions which help to lessen the impact of various assaults. The fruitful conveyance of message with the capacity to scatter and evasion of broken connections is more dependable than standard verified information transmission instrument. We created and reproduced a structure for the recognition of specific sending assaults utilizing MANET advancements. A system situation is conveyed and a few tests were performed for the check and approval of the proposed arrangement. Future work is bearing to trim down the proportion of End to End delays is Improve the Recitation of The System New Way.

\section{REFERENCES}

[1] Silva, M.M., Subramanian, A., Ochi, L.S. (2015). An iterated local search heuristic for the split delivery vehicle routing problem. Computers \& Operations Research, 53: 234-249. https://doi.org/10.1016/j.cor.2014.08.005

[2] Belengue, J.M., Martinez, M.C., Mota, E. (2005). A lower bound for the split delivery vehicle routing problem. Operations Research, 48(5): 801-810. https://doi.org/10.1287/opre.48.5.801.12407

[3] Lee, C.G., Epelman, M.A., White, C.C., Bozer, Y.A. (2006). A shortest path approach to the multiple- vehicle routing problem with split pick-ups. Transportation Research B, 40: 265-284. https://doi.org/10.1016/j.trb.2004.11.004

[4] Dror, G.L.M., Trudeau, P. (1994). Vehicle routing with split deliveries. Discrete Applied Mathematics, 50: 239254. https://doi.org/10.1016/0166-218X(92)00172-I

[5] Jin, M.Z., Liu, K., Bowden, R.O. (2007). A two-stage algorithm with valid inequalities for the split delivery vehicle routing problem. International Journal of Production Economics, 105(105): 228-242. https://doi.org/10.1016/j.ijpe.2006.04.014

[6] Archetti, C., Bianchessi, N., Speranza, M.G. (2011). A column generation approach for the split delivery vehicle routing problem. Networks, 58(4): 241-254. https://doi.org/10.1002/net.20467

[7] Gulczynski, D., Golden, B., Wasil, E. (2010). The split delivery vehicle routing problem with minimum delivery amounts. Transportation Research Part E: Logistics and Transportation Review, 46(5): 612-626. https://doi.org/10.1016/j.tre.2009.12.007

[8] Tang, J., Ma, Y., Guan, J., Yan, C. (2013). A max-min ant system for the split delivery weighted vehicle routing problem. Expert Systems with Applications, 40(18): 7468-7477. https://doi.org/10.1016/j.eswa.2013.06.068

[9] Boudia, M., Prins, C., Reghioui, M. (1976). An effective memetic algorithm with population management for the split delivery vehicle routing problem. Hybrid Metaheuristics, 16-30. https://doi.org/10.1007/978-3540-75514-2_2

[10] Glover, F. (1992). New ejection chain and alternating path methods for traveling salesman problems. Computer Science and Operations Research, 18: 491-507. http://doi.org/10.1016/B978-0-08-040806-4.50037-X

[11] Rego, C. (2001). Node-ejection chains for the vehicle routing problem: Sequential and parallel algorithm. Parallel Computing, 27: 201-222. 
https://doi.org/10.1016/S0167-8191(00)00102-2

[12] Chen, P., Golden, B., Wang, X., Wasil, E. (2017). A novel approach to solve the split delivery vehicle routing problem. International Transactions in Operational Research, 24(12):

27-41.

https://doi.org/10.1111/itor.12250

[13] Dror, M., Trudeau, P. (1989). Savings by split delivery routing. Transportation Science, 23(3): 141-145. https://doi.org/10.1287/trsc.23.2.141

[14] Archetti, C., Savelsbergh, M.W.P., Speranza, M.G. (2006). Worst-case analysis for split delivery vehicle routing problems. Transportation Science, 40(2): 226234. https://doi.org/10.1287/trsc.1050.0117

[15] Archetti, C., Speranza, M.G., Hertz, A. (2006). A tabu search algorithm for the split delivery vehicle routing problem. Transportation Science, 40(1): 64-73. https://doi.org/10.1287/trsc.1040.0103

[16] Chen, S., Golden, B., Wasil, E. (2007). The split delivery vehicle routing problem: Applications, algorithms, test problems, and computational results. Networks, 49(4): 318-329. https://doi.org/10.1002/net.20181

[17] Qiu, M., Fu, Z. (2018). A tabu search algorithm for the discrete split delivery vehicle routing problem. Journal of Harbin Engineering University. https://doi.org/23.1390.U.20180804.1217.004.html

[18] Yellow, P. (1970). A computional modification to the savings method of the vehicle routing problem. European Journal of Operational Research, 21: 281-283. 